\title{
Influence of Asymmetric Energetic Ion Distributions on Sawtooth Stabilization*
}

\author{
Jonathan P. Graves \\ Centre de Recherches en Physique des Plasmas, Association EURATOM-Confédération Suisse, \\ EPFL, 1015 Lausanne, Switzerland
}

(October 13, 2003)

\begin{abstract}
The effect of energetic asymmetrically distributed ions on the stability of the internal kink mode in tokamaks is analyzed. Circulating ions which intersect the resonant surface due to finite radial excursion contribute to the mode either in the region of favorable or unfavorable curvature depending on the sign of $v_{\|}$. Internal kink mode stabilization for predominantly co-circulating ion populations is consistent with the observation of long sawteeth using tangential co-injection of neutral beams in JT-60U [G. J. Kramer et al, Nucl. Fusion 40, 1383 (2000)]. Off-axis neutral beam heating emerges as a possible means of assisting sawtooth control in future fusion grade experiments.

53.35.Py, 52.55.Fa, 52.55.Tn
\end{abstract}

Typeset using REVTEX

*Submitted to Physical Review Letters 
Magnetohydrodynamic (MHD) stability of plasmas in the presence of energetic ions is a crucial issue for present and future large tokamak experiments. Such ions include $3.5 \mathrm{MeV}$ alpha particles, produced naturally in deuterium-tritium (DT) fusion reactions, and energetic minority ions produced by auxiliary heating methods such as neutral beam injection (NBI) and ion cyclotron resonance heating (ICRH). A fraction of the energetic ions which have a relatively small velocity component parallel to the magnetic field lines are trapped in the low magnetic field strength region or outer region of tokamaks. Such magnetically trapped energetic ions have been shown [1] to stabilize a key core instability known as the sawtooth, thereby lengthening the period between sequential soft-x-ray relaxations [2]. In future large scale fusion devices the trapped fraction of alpha particles could stabilize sawteeth for many confinement times [3] such that the eventual relaxation event could give rise to an undesirable loss of energy confinement and loss of particles in the core including alpha particles. Recent observations [4] linking the sawtooth free period with the early onset of confinement degrading neo-classical tearing modes (NTM's) especially highlights the importance of exploring techniques to control sawteeth.

The stabilizing role of ICRH energetic trapped ions on sawteeth has received continued interest within the last two decades [1]. In contrast, the negative ion-based neutral beam injection (NNBI) planned for the International Tokamak Experimental Reactor (ITER) [5] is expected to yield a highly energetic population of ions (up to $1 \mathrm{MeV}$ ) with a small trapped fraction. Nevertheless such a scenario employed in JT-60U [6] with $350 \mathrm{keV}$ ions demonstrates significant sawtooth stabilization where the circulating (untrapped) ions predominantly propagate in the same (co) direction as the plasma current. This letter explores the effect of such asymmetrically distributed circulating ions on the $m=1, n=1$ (here $m$ and $n$ are the poloidal and toroidal mode numbers) internal kink mode, the ideal stability of which is widely regarded as a fundamental component of the mechanism that triggers sawteeth. This is particularly valid in the presence of an energetic minority ion population which typically gives rise to a large perturbed potential energy $\delta W[3]$. One important consequence of the self consistent treatment employed in the present letter is that the effect of 
finite radial excursion of particles contained completely within the $q=1$ surface yields an adiabatic contribution which nullifies the recently discovered non-adiabatic term of Wang et al. [7]. Nevertheless it is found that an important $\delta W$ contribution survives via ions which intersect the $q=1$ surface during their poloidal orbits. Whether these ions provide a sink or source of free energy depends on the direction of injection and the sign of the energetic ion pressure gradient at the resonant surface. In addition to providing an explanation for sawtooth stabilization in JT-60U, the unexpected yet crucial finite orbit effects described here indicate the possible use of both on- and off-axis neutral beam heating to assist the control of sawteeth in fusion grade plasmas.

The plasma consists of isotropic bulk ions and electrons, which collectively form the core ' $c$ ' plasma, and energetic or hot ' $h$ ' ions. The relevant ordering employed is $\beta_{h} \sim \beta_{c} \sim O\left(\epsilon^{2}\right)$, where $\beta_{h, c}=2 \mu_{0} P_{h, c} / B_{0}^{2}$ is the ratio of hot or core particle pressure and the magnetic pressure, and $\epsilon$ is the inverse aspect ratio of the device. The response of energetic ions to the internal kink mode is obtained by perturbing the hot ion distribution $F_{h}\left(\mathcal{E}, \mathcal{P}_{\phi}, \mu\right)$ with respect to the constants of motion $\mathcal{E}=v^{2} / 2, \mu=v_{\perp}^{2} / 2$ and $\mathcal{P}_{\phi}=R v_{\phi}+Z e \psi / m_{h}$, where in circular cross section geometry $\psi$ is related to the minor radius $r$ by $r B_{0} d r=q(r) d \psi$. Here, the magnetic field strength $B=B_{0}(1-\epsilon \cos \theta)$ with $\theta$ the poloidal angle, $q$ the safety factor, $\epsilon=r / R_{0}$ and $R=R_{0}(1-\epsilon \cos \theta)$ is the major radius. Focusing on ideal MHD modes in a low $\beta$ and small $\epsilon$ tokamak yields [8] the perturbed distribution function $\delta F_{h}=\delta F_{h f}+\delta F_{h k}$, where $\delta F_{h f}=-\left(Z e / m_{h}\right)(\boldsymbol{\xi} \cdot \nabla \psi) \partial F_{h} / \partial \mathcal{P}_{\phi}$ is the adiabatic (fluid) contribution with $\boldsymbol{\xi} \sim \exp (-i \theta-i \phi-i \omega t)$ the MHD internal kink fluid displacement, and the non-adiabatic (kinetic) contribution $\delta F_{h k}$ can be approximately written as the solution to the drift kinetic equation:

$$
\left\{\omega+\dot{\phi}+i \dot{\chi} \frac{\partial}{\partial \chi}\right\} \delta F_{h k}=-\left(\omega-\omega_{* h}\right) \frac{\partial F_{h}}{\partial \mathcal{E}}\left(v_{\|}^{2}+\frac{v_{\perp}^{2}}{2}\right) \boldsymbol{\xi}_{\perp} \cdot \boldsymbol{\kappa},
$$

where $\dot{\chi} \partial / \partial \chi=\dot{\theta} \partial / \partial \theta+\dot{r} \partial / \partial r, \omega_{* h}=\partial F_{h} / \partial \mathcal{P}_{\phi}\left(\partial F_{h} / \partial \mathcal{E}\right)^{-1}$ is the diamagnetic frequency and $\boldsymbol{\kappa}$ the magnetic curvature vector. Equation (1) can be readily solved for circulating particles as a Fourier expansion in $\chi$ harmonics upon employing the boundary condition 
$\delta F_{h}\left(\chi=0, \pm v_{\|}\right)=\delta F_{h}\left(\chi=2 \pi, \pm v_{\|}\right)$and limiting the orbit width $\Delta_{b} / r_{1} \sim \epsilon_{1}$ (where $\epsilon_{1}=r_{1} / R_{0}$ and $\left.q\left(r_{1}\right)=1\right)$ to give $\delta F_{h k}=\sum_{m=-\infty}^{\infty} \delta F_{h k}^{(m)} \exp (-i m \chi-i \phi-i \omega t)$ with:

$$
\delta F_{h k}^{(m)}=-\frac{\left(\omega-\omega_{* h}\right)}{\omega-\left\langle\omega_{d}\right\rangle+\dot{\chi}[m-q(\bar{r})]} \frac{\partial F_{h}}{\partial \mathcal{E}}\left\langle\left(v_{\|}^{2}+\frac{v_{\perp}}{2}\right) \boldsymbol{\kappa} \cdot \hat{\boldsymbol{\xi}}_{\perp} \exp (i m \chi)\right\rangle
$$

where $\boldsymbol{\xi}_{\perp}=\hat{\boldsymbol{\xi}}_{\perp} \exp (-i \phi-i \omega t),\langle y\rangle=(\oint d \chi y / \dot{\chi}) / \oint d \chi / \dot{\chi}, \bar{r}$ is the mean particle radius and $\omega_{d}$ is the toroidal magnetic drift frequency. For simplicity the energetic ions are henceforth assumed to be purely circulating so that $v_{\|}^{2}=2 \mathcal{E}$ is conserved and $v_{\perp}=0$. Following Ref. [9] by employing a right handed system $(r, \theta, \zeta=-\phi)$ the conservation of $\mathcal{P}_{\phi}$ yields particle trajectories $r=\bar{r}+\sigma \Delta_{b} \cos \theta, \theta=\chi-\sigma(1+s)\left(\Delta_{b} / \bar{r}\right) \sin \chi, \zeta=q(\bar{r}) \chi$ with $\chi(t)-\chi\left(t^{\prime}\right)=$ $\sigma \omega_{b}\left(t-t^{\prime}\right), \omega_{b}=\left|v_{\|}\right| / q(\bar{r}) R, \Delta_{b}=\left|v_{\|}\right| q(\bar{r}) / \Omega_{c}, \Omega_{c}=e Z B_{0} / m_{h}, s=d \log q / d \log r$ and $\sigma=\operatorname{sign}\left(v_{\|}\right)=\operatorname{sign}\left(-v_{\phi}\right)$ for which in the following analysis $\sigma=1$ corresponds to cocirculating ions and $\sigma=-1$ counter circulating ions. Hence, noting that $\dot{\zeta}=\sigma\left|v_{\|}\right| / R$ then from Eq. $(2), \delta F_{h k}^{(0)}$ can be identified with the non-adiabatic sideband response described in detail in Ref. [7]. The only other non-negligible harmonic in Eq. (2) is the $m=1$ fundamental $\delta F_{h k}^{(1)}$ discovered in Ref. [10]. This finite orbit term is the non-adiabatic response of particles which intersect the $q=1$ radius and is retained in the present letter.

Closed forms for the energetic particle contributions are obtained by identifying $\boldsymbol{\xi}_{\perp}$ with the leading order solution to the Euler-Lagrange equation for the internal kink in the absence of energetic particles: $\hat{\xi}_{r}=\xi_{0} \mathrm{H}\left[r_{1}-r\right] \exp (-i \theta)$ and $\hat{\xi}_{\theta}=-i \hat{\xi}_{r}$, with $\mathrm{H}$ the Heavy-side step function. Note that the use of a Heavy-side step function with discontinuity at $r_{1}$ is valid for the treatment of NNBI ions close to and intersecting the $q=1$ surface because $\Delta_{b} / r_{1} \sim \epsilon_{1}$, while the characteristic width of the transition layer $\delta \sim \epsilon_{1}^{2} r_{1}$. Hence one obtains $\boldsymbol{\kappa} \cdot \hat{\boldsymbol{\xi}}_{\perp}=-\left(\xi_{0} / R\right) \mathrm{H}\left[r_{1}-\bar{r}-\sigma \Delta_{b} \cos \chi\right]$. Taking into account the finite radial and poloidal drift gives the fluid contribution:

$$
\delta \hat{F}_{h f}=-\frac{\Omega_{c} \bar{r}}{q(\bar{r})} \xi_{0} \mathrm{H}\left[r_{1}-\bar{r}-\sigma \Delta_{b} \cos \chi\right] \frac{\partial F_{h}}{\partial \mathcal{P}_{\phi}}\left[\exp (-i \chi)+\sigma \frac{\Delta_{b}}{\bar{r}}\right]
$$

where $\delta F=\hat{\delta F} \exp (-i \phi-i \omega t)$. Meanwhile for the sawtooth relevant limit $\omega \ll\left\{\dot{\chi}\right.$ and $\left.\omega_{* h}\right\}$ and for the limit $\left\langle\omega_{d}\right\rangle \ll \dot{\chi}$, which applies to energetic circulating ions, one can show that the 
second term in Eq. (2) can be written as $\delta F_{h k}^{(0)}=\Omega_{c} \xi_{0} \mathrm{H}\left[r_{1}-\bar{r}-\sigma \Delta_{b} \cos \chi\right] \sigma\left(\Delta_{b} / q\right) \partial F_{h} / \partial \mathcal{P}_{\phi}$. This $m=0$ non-adiabatic contribution [7] is therefore seen to scale as a finite orbit term and is exactly cancelled by the second term in Eq. (3). A further finite orbit contribution, describing the adiabatic response to the mode of particles intersecting the $q=1$ surface, is contained in the Heavy-side step function in the first term of Eq. (3). This finite orbit term does not disappear. However, to correctly account for the toroidal [11] and anisotropic [12] contributions that energetic ion populations make with ordering $\beta_{h} \sim \beta_{c}$, it is more convenient to express $\delta F_{h f}$ in terms of an expansion around $r$ instead of $\bar{r}$ :

$$
\delta \hat{F}_{h f}=-\xi_{0} H\left[r_{1}-r\right]\left[\exp (-i \theta)-\sigma \frac{\Delta_{b}}{2 r}\left(1+\exp (-i 2 \theta)\left(1-r \frac{\partial}{\partial r}\right)\right] \frac{\partial F_{h}(r)}{\partial r} .\right.
$$

Cancellation with the non-adiabatic term of Ref. [7] is then made when evaluating the energetic ion contributions to the perturbed potential energy

$$
\delta W_{h}=\frac{m_{h}}{2} \int d^{3} x \int d^{3} v v_{\|}^{2} \boldsymbol{\kappa} \cdot \boldsymbol{\xi}_{\perp}^{*} \delta F_{h}
$$

where $d^{3} v=2^{1 / 2} \pi \sum_{\sigma} \int_{0}^{\infty} d \mathcal{E} \mathcal{E}^{1 / 2} B \int_{0}^{\infty} d \lambda, \lambda=\mu / \mathcal{E}$ and $d^{3} x=2 \pi\left(R^{2} / R_{0}\right) d \theta d r r$ and one may use $d \theta d r r=\Omega_{c}^{-1} q(\bar{r}) d \chi d \mathcal{P}_{\phi}$. As mentioned above it is sufficient to use only $\delta \hat{F}_{h}=$ $\hat{F}_{h f}+\delta F_{h k}^{(0)}+\delta F_{h k}^{(1)} \exp (-i \chi)$ in Eq. (5).

Since all the energetic particles stream along field lines $(\mu=0)$ then the pressure is anisotropic. The components of the pressure tensor $\left\{P_{h \|}, P_{h \perp}\right\}=m_{h} \int d^{3} v F_{h}\left\{v_{\|}^{2}, v_{\perp}^{2} / 2\right\}$ are $P_{h \|}=\left\langle P_{h \|}\right\rangle(1-\epsilon \cos \theta)$ and $P_{h \perp}=0$ for which $P_{h} \equiv\left(P_{h \|}+P_{h \perp}\right) / 2=P_{h \|} / 2$ and $\langle P\rangle \equiv(2 \pi)^{-1} \int_{0}^{2 \pi} d \theta P$. Hence, inserting the first term in Eq. (4) into Eq. (5) and changing the order of differentiation yields a non-finite orbit term, or $O\left(\left(\Delta_{b} / r_{1}\right)^{0}\right)$ contribution, which combines with the core MHD contribution as:

$$
\begin{aligned}
& \delta W_{f}\left[O\left(\left(\Delta_{b} / r_{1}\right)^{0}\right)\right]=\frac{1}{2} \int d^{3} x\left\{\frac{\delta B_{\perp}^{2}}{\mu_{0}}+\frac{B^{2}}{\mu_{0}}\left(\boldsymbol{\nabla} \cdot \boldsymbol{\xi}_{\perp}+2 \boldsymbol{\xi}_{\perp} \cdot \boldsymbol{\kappa}\right)^{2}\right. \\
& \left.-2\left[\boldsymbol{\xi}_{\perp} \cdot \boldsymbol{\nabla}\langle P\rangle\right]\left(\boldsymbol{\kappa} \cdot \boldsymbol{\xi}_{\perp}^{*}\right)-j_{\|}\left(\boldsymbol{\xi}_{\perp}^{*} \times \hat{\boldsymbol{e}}_{\|}\right) \cdot \boldsymbol{\delta} \boldsymbol{B}_{\perp}\right\}-2 \pi^{2} \xi_{0}^{2} \int_{0}^{r_{1}} d r r \epsilon \frac{d}{d r}\left\langle P_{h}\right\rangle,
\end{aligned}
$$

where $\langle P\rangle=\left\langle P_{h}\right\rangle+P_{c}$ is the total flux averaged pressure. Hence the last term in Eq. (6) is the anisotropic correction to the isotropic MHD terms. The latter is henceforth equated 
with Bussac's [11] toroidal ' $T$ ' term $\delta W_{T}$, which for a simple quadratic $q$ profile is given by $\delta \hat{W}_{T} \approx 3\left(1-q_{0}\right)\left(0.3^{2}-\beta_{p}^{2}\right)$, where $q_{0}=q(r=0), \beta_{p}=-\left(2 \mu_{0} / B_{0}^{2} \epsilon_{1}^{2}\right) \int_{0}^{r_{1}} d r\left(r / r_{1}\right)^{2} d\langle P\rangle / d r$ and $\delta \hat{W}=\delta W /\left(2 \pi^{2} \xi_{0}^{2} \epsilon_{1}^{4} R_{0} B_{0}^{2} / \mu_{0}\right)$. Cancellation of the potential energy term associated with $\delta f_{h k}^{(0)}$ is seen upon substituting the finite orbit terms of Eq. (4) into Eq. (5) and integrating by parts. One is then left with only the boundary terms at $q=1$. Hence the sum of the kinetic and finite orbit fluid terms are

$$
\delta W_{h k}+\delta W_{h f}\left[O\left(\Delta_{b} / r_{1}\right)\right]=\left.\frac{2^{2} \pi^{3} m_{h} \xi_{0}^{2}}{\Omega_{c}} \int_{0}^{\infty} d \mathcal{E} \mathcal{E}^{2} \sum_{\sigma}\left(\frac{2 g(l)}{\pi^{2} s_{1}}-\sigma\right) r_{1} \frac{\partial \tilde{F}_{h}}{\partial r}\right|_{r_{1}}
$$

where the total internal kink potential energy $\delta \hat{W}$ is the sum of Eqs. (6) and (7). Here $F_{h}=\tilde{F}_{h}(r, \mathcal{E}, \sigma) \delta(\lambda) / B_{0}$ and $g(l)=2 l+\left(l^{2}-1\right) \ln [(l-1) /(l+1)]$ with $l=\left(\omega-\left\langle\omega_{d}\right\rangle\right) / \omega_{s}$ and $\omega_{s}=s_{1} 2 \mathcal{E} /\left(r_{1} R_{0} \Omega_{c}\right)$. The first and second terms in Eq. (7) are recognized as the kinetic and fluid response to the internal kink mode of particles intersecting the $q=1$ radius. The fluid term is seen to depend directly on the direction of injection, and cancels for a symmetric distribution. The kinetic term has both reactive (real) and dissipative (imaginary) components which depend weakly on asymmetry through the magnetic drift $\left\langle\omega_{d}\right\rangle[13]$. One important point is that Eq. (7) depends only on plasma parameter values at $q=1$. Thus the effect of tangential NBI on sawteeth is expected to be strongly dependent on the heating deposition relative to $r_{1}$.

For the examining the case of tangential NBI it is sufficient to employ a slowing down distribution function of the form:

$$
F_{h}=\frac{\left\langle P_{h}\right\rangle_{\sigma} H\left[\mathcal{E}^{I}-\mathcal{E}\right]}{2^{1 / 2} \pi m_{h} B_{0} \mathcal{E}^{I}} \mathcal{E}^{-3 / 2} \delta(\lambda)
$$

where $\mathcal{E}^{I}$ is the injection energy and $\left\langle P_{h}\right\rangle_{+1}$ and $\left\langle P_{h}\right\rangle_{-1}$ are the respective pressures from particles flowing with $v_{\|}>0$ and $v_{\|}<0$. Hence $\left\langle P_{h}\right\rangle=\left\langle P_{h}\right\rangle_{+1}+\left\langle P_{h}\right\rangle_{-1}$, and one can also define an angle of asymmetry

$$
A=\frac{\left\langle P_{h}\right\rangle_{+1}-\left\langle P_{h}\right\rangle_{-1}}{\left\langle P_{h}\right\rangle}
$$

Hence $A=1$ for pure co-injection, $A=-1$ for pure counter-injection, and $A=0$ for 
balanced injection. The distribution of Eq. (8) yields the following expression for the total normalized potential energy:

$$
\delta \hat{W}=\delta \hat{W}_{T}+\frac{1}{2} \beta_{p h}+\left.\frac{1}{3} \epsilon_{1}^{-1}\left(\frac{\Delta_{b}^{I}}{r_{P h}}\right) \tilde{\beta}_{p h}\left(A-\frac{2 F[\hat{\Omega}]}{\pi s_{1}}\right)\right|_{r_{1}}
$$

where a 'signed' scale length for the hot pressure variation is defined $r_{P h}=-\left.\left(d\left\langle P_{h}\right\rangle / d r\right)^{-1}\left\langle P_{h}\right\rangle\right|_{r_{1}}, \beta_{p h}=-\left(2 \mu_{0} / B_{0}^{2} \epsilon_{1}^{2}\right) \int_{0}^{r_{1}} d r\left(r / r_{1}\right)^{2} d\left\langle P_{h}\right\rangle / d r$, $\tilde{\beta}_{p h}=2 \mu_{0}\left\langle P_{h}\left(r_{1}\right)\right\rangle /\left(\epsilon_{1}^{2} B_{0}^{2}\right)$ and $F(\hat{\Omega})$ is approximately given by Eq. (15) of Ref. [14], with $\hat{\Omega}=\omega / \omega_{s}^{I}$, where superscript $I$ denotes values taken at the injection energy. The definition of $F[\hat{\Omega}]$ given in Ref. [14] is valid for $\left\langle\omega_{d}\right\rangle \ll \omega_{s}$, which is appropriate because $\left\langle\omega_{d}\right\rangle \sim\left(\epsilon_{1} / s_{1}\right) \omega_{s}$ at $r_{1}$. For low frequency sawtooth-type modes it is typically found that $\Omega \ll 1$, and given that $\Re\{F[0]\}=0$, it is clear that the reactive part of Betti's contribution [14] (the real part of the fourth term in Eq. (9)) is, for an asymmetric distribution, small compared to the finite orbit fluid term (third term in Eq. (9)). The first and second terms of Eq. (9) are respectively the toroidal and anisotropic contributions to stability.

The marginal stability problem is now examined for the parameters of NNBI sawtoothing plasmas in JT-60U. Obtained is the poloidal beta for the core plasma $\beta_{p c}$ which satisfies the dispersion relation $\sqrt{3} \gamma_{I} / \omega_{A}=-\epsilon_{1}^{2} \delta \hat{W} / s_{1}$, where $\gamma_{I}^{2}=-\omega\left(\omega-\omega_{* i}\right)$, with marginal condition $\gamma \equiv \Im\{\omega\}=0$. Here $\omega_{* i} \approx 1 \times 10^{4} \mathrm{rad} / \mathrm{s}$ is the ion diamagnetic frequency and $\omega_{A}=v_{A} / R_{0} \approx 4 \times 10^{6} \mathrm{rad} / \mathrm{s}$, with $v_{A}$ the Alfvén velocity. Given that $r_{1} \approx 0.3 \mathrm{~m}, R_{0}=3.2$ $\mathrm{m}, B_{0}=3.5 \mathrm{~T}$ and an injection energy of $350 \mathrm{keV}$, one finds $\Delta_{b}^{I}=0.035 \mathrm{~m}$ and therefore $\epsilon_{1}^{-1} \Delta_{b}^{I} / r_{1} \sim 1$. Hence all the terms in Eq. (9) are of a similar order of magnitude if $\left|r_{P h}\right| \sim r_{1}$. A safety factor profile of the form $q(r)=q_{0}\left(1+2.8(r / a)^{2}\right)^{1 / 2}$ is chosen with $q_{0}=0.8$ and $a=1 \mathrm{~m}$ the minor radius. This gives $\epsilon_{1}=0.094$ and $s_{1}=0.4$. Finally, the energetic ion pressure profile is taken to be of the form $\left\langle P_{h}\right\rangle=P_{0}\left(1-\left[\left(r-r_{b}\right) / \Delta\right]^{2}\right)^{\nu}$, with $P_{0}$ a constant and in all cases $\nu=4$ is employed. Simulation of JT-60U on-axis heating [6] is undertaken with $r_{b}=0, \Delta=a$ and $\tilde{\beta}_{p h}=0.41$ so that $\beta_{p h}=0.09$ and $r_{P h}=1.83 r_{1}$. Also examined is the case of off-axis heating with $r_{b}=\Delta=0.5 a$ and $\tilde{\beta}_{p h}=0.15$ which gives $\beta_{p h}=-0.085$ and $r_{P h}=-0.87 r_{1}$. 
The variation of $\beta_{p c}$ with injection asymmetry $A$ for JT-60U on-axis heating parameters [6] is given by the solid curve of Fig. 1. For the pure co-injection case $(A=1)$, where all the energetic ions stream along magnetic field lines, $\beta_{p c}=0.4$ is approximately the value attained in JT-60U prior to a sawtooth crash. This is a large increase over the marginal value calculated in the absence of energetic ions: $\beta_{p}=\beta_{p c}=0.2$. For balanced injection the finite orbit fluid contribution is zero, but energetic ions slightly modify $\beta_{p c}$ through their contribution to toroidicity [11], anisotropy [12] and finite orbit kinetic effects [10]. The stabilizing effect of anisotropy dominates so that the total marginal poloidal beta is raised to $\beta_{p}=0.23$, and hence $\beta_{p c}=\beta_{p}-\beta_{p h}=0.14$. The finite orbit fluid response is strongly destabilizing for negative $A$. In particular, for $A<-0.27$ a positive $\beta_{p c}$ satisfying $\gamma=\Im\{\omega\}=0$ does not exist. Hence it is predicted that discharges employing on-axis counter injection with a centrally peaked pressure profile will exhibit much shorter sawtooth free periods that with on-axis co-injection [6].

The dashed line in Fig. 1 is relevant for off-axis heating where the peak in the energetic pressure profile is outside the $q=1$ surface. Such profiles can be obtained in high density plasmas. Examination of the new finite orbit fluid term clearly shows that a positive pressure gradient (negative $r_{P h}$ ) at $r_{1}$ gives rise to a $\beta_{p c}$ dependence on $A$ which is opposite to that described above with centrally peaked heating. Unlike the toroidal [11] and anisotropic [12] contributions, which depend on radially integrated quantities, the sign and magnitude of the pressure gradient at the $q=1$ surface is crucial for the finite orbit terms. It is therefore expected that in sufficiently high density plasmas, co-injection could be employed to shorten sawteeth and counter injection could be employed to lengthen sawteeth.

The response of unbalanced tangentially injected energetic ions can be compared with the trapped ion kinetic response of an isotropic alpha particle population to the internal kink mode. For fusion plasma applications the latter contribution $\delta \hat{W}_{\alpha}$ can be added to the terms in Eq. (9) together also with the collisionless response of trapped thermal ions (e.g. Ref. [15]). The finite orbit fluid contribution, identified with the third term in Eq. (9), scales with $\delta \hat{W}_{\alpha}$ as: 


$$
\frac{\delta \hat{W}_{h f}\left[O\left(\Delta_{b} / r_{1}\right)\right]}{\delta \hat{W}_{\alpha}}=\frac{\pi}{3}\left(\frac{2}{\epsilon_{1}}\right)^{1 / 2} A\left(\frac{\Delta_{b}^{I}}{r_{P h}}\right) \frac{\tilde{\beta}_{p h}}{\beta_{p \alpha}}
$$

where $\beta_{p \alpha}=-\left(2 \mu_{0} / B_{0}^{2} \epsilon_{1}^{2}\right) \int_{0}^{r_{1}} d r\left(r / r_{1}\right)^{3 / 2} d P_{\alpha} / d r$. For the $1 \mathrm{MeV}$ NNBI heating system planned for ITER [5] one finds $\Delta_{b}^{I} / r_{1} \approx \epsilon_{1} / 2$. Given the latter scaling, Eq. (10) indicates that off-axis co-injection (with $r_{b}>r_{1}, r_{P h}<0$ and $A \approx 1$ ) could be employed as a possible means of competing against the strongly stabilizing energetic ion response of alpha particles and thereby assist sawtooth control if $\tilde{\beta}_{p h} /\left|r_{P h}\right| \sim \epsilon_{1}^{-1 / 2} \beta_{p \alpha} / r_{1}$.

This letter provides an explanation for the sawtooth stabilization experiments in JT-60U [6] involving the finite orbit effect of circulating particles that intersect the $q=1$ surface during their poloidal orbits. Such particles contribute either in the region of favorable or unfavorable curvature for respectively toroidally co-circulating or counter-circulating ions. Other finite orbit effects also exist in the adiabatic response of energetic circulating ions to the internal kink mode, but these serve to nullify the sideband $m=0$ non-adiabatic response of asymmetric circulating ions recently discovered in Ref. [7]. Depending on the choice of toroidal direction and radial deposition of tangentially injected beam ions, a mechanism is discovered which could enable energetic ion destabilization of sawteeth. Such scenarios will be of particular interest in future efforts to control sawteeth in plasmas with large fusion alpha populations.

The author would like to acknowledge useful discussions with S. Günter, R. J. Hastie and O. Sauter. This work was partly funded by the Fonds National Suisse de la Recherche Scientifique. 


\section{REFERENCES}

[1] D. J. Campbell, D. F. H. Start, J. A. Wesson, D. V. Bartlett, et al., Phys. Rev. Lett. 602148 (1988); R. B. White, P. H. Rutherford, P. Colestock and M. N. Bussac, Phys. Rev. Lett. 60, 2038 (1988); B. Coppi, P. Detragiache, S. Migliuolo, F. Pegoraro and F. Porcelli, Phys. Rev. Lett. 63, 2733 (1989); F. Porcelli, Plasma Phys. Controlled Fusion 33, 1601 (1991); J. P. Graves, et al Phys. Rev. Lett. 84, 1204 (2000).

[2] S. von Goeler, W. Stodiek and N. Sauthoff, Phys. Rev. Lett. 33, 1201 (1974).

[3] F. Porcelli, D. Boucher and M. N. Rosenbluth, Plasma Phys. Controlled Fusion 38, $2163(1996)$.

[4] O. Sauter et al., Phys. Rev. Lett. 88, 105001 (2002).

[5] ITER Physics Basis Editors et al., Nucl. Fusion 39, 2137, (1999).

[6] G. J. Kramer et al., Nucl. Fusion 40, 1383 (2000).

[7] S. Wang et al., Phys. Rev. Lett. 88, 105004 (2002).

[8] P. Helander et al., Phys. Plasmas 4, 2182 (1997).

[9] J. D. Meiss and R. D. Hazeltine, Phys. Fluids B 2, 2563 (1990).

[10] R. Betti and J. P. Freidberg, Phys. Rev. Lett. 70, 3428 (1993).

[11] M. N. Bussac, R. Pellat, D. Edery and J. L. Soulé, Phys. Rev. Lett. 35, 1638 (1975).

[12] A. B. Mikhailovski, Sov. J. Plasma Phys. 9, 190 (1983); J. P. Graves, N. N. Gorelenkov and O. Sauter, Phys. Plasmas 10, 1034 (2003).

[13] Ya. I. Kolesnichenko, Phys. Plasmas 10, 1449 (2003).

[14] R. Betti, Phys. Plasmas Control. Fusion 35, 941 (1993).

[15] J. P. Graves, R. J. Hastie and K. I. Hopcraft, Plasma Phys. Contol. Fusion 42, 1049 (2000). 


\section{FIGURES}

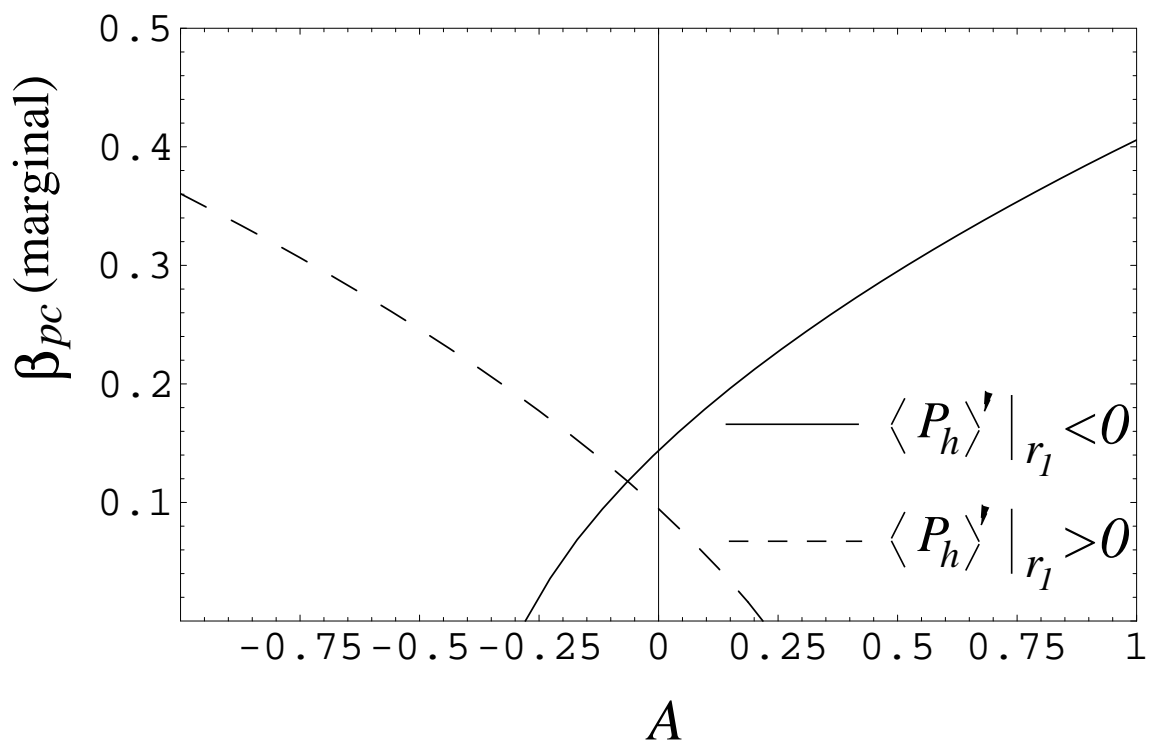

FIG. 1. Showing the marginal poloidal beta for the core plasma $\beta_{p c}$ as a function of injection asymmetry $A$ for two different energetic ion pressure profiles. The solid curve is for central heating $\left(r_{b}=0\right)$ for which $A=1$ corresponds to the pure co-injection scenario in JT-60U. The dashed curve is for $r_{b}=a / 2$, such that the heating profile is peaked outside $r_{1}$. 